\title{
Trace Amounts of Antibiotic Altered Metabolomic and Microbial Profiles of Weaned Pigs Infected with a Pathogenic E. coli
}

\section{Kwangwook Kim}

University of California Davis https://orcid.org/0000-0001-5854-6047

\section{Cynthia Jinno}

University of California Davis

Peng Ji

University of California Davis

Yanhong Liu ( $\square$ yahliu@ucdavis.edu )

University of California https://orcid.org/0000-0001-7727-4796

\section{Research}

Keywords: carbadox, colon microbiota, enterotoxigenic Escherichia coli, metabolomics, weaned pigs

Posted Date: November 11th, 2021

DOI: https://doi.org/10.21203/rs.3.rs-1061523/v1

License: (a) (i) This work is licensed under a Creative Commons Attribution 4.0 International License. Read Full License 


\section{Abstract}

Background: Our previous study has shown that supplementation of trace amounts of antibiotic exacerbated the detrimental effects of enterotoxigenic $E$. coli (ETEC) infection and delayed the recovery of pigs that may be associated with modified metabolites and metabolic pathways. Therefore, the objective of this study was to explore the impacts of trace levels of antibiotic (carbadox) on host metabolic profiles and colon microbiota of weaned pigs experimentally infected with ETEC F18.

Results: The multivariate analysis highlighted a distinct metabolomic profile of serum and colon digesta between trace amounts of antibiotic (TRA) and label-recommended dose antibiotic (REC) on d 5 postinoculation (PI). The relative abundance of metabolomic markers of amino acids, carbohydrates, and purine metabolism were significantly differentiated between the TRA and REC groups $(q<0.2)$. In addition, pigs in REC group had the highest $(P<0.05)$ relative abundance of Lactobacillaceae and tended to have increased $(P<0.10)$ relative abundance of Lachnospiraceae in the colon digesta on $\mathrm{d} 5 \mathrm{PI}$. On d $11 \mathrm{PI}$, pigs in REC had greater $(P<0.05)$ relative abundance of Clostridiaceae compared with other groups, whereas had reduced $(P<0.05)$ relative abundance of Prevotellaceae than pigs in control group.

Conclusions: Trace amounts of antibiotic resulted in differential metabolites and metabolic pathways that may be associated with its slow responses against ETEC F18 infection. The altered gut microbiota profiles by label-recommended dose antibiotic may contribute to the promotion of disease resistance in weaned pigs.

\section{Background}

Trace amounts of antibiotics are emerging contaminants of environmental concern due to their potential risks on non-target organisms and the spread of bacterial resistance [1]. The excessive and imprudent use of human and veterinary antibiotics significantly contributes to a continuous release of antibiotics into the environment, thus, a variety of trace concentrations of antibiotics have been detected in surface water, wastewater, soil, air, and dust [2-5]. Exposure to trace levels of antibiotics may cause unexpected adverse effects on the host, such as allergic reactions, disruption of digestive system function, and shaping the metabolites and microbial community $[6,7]$. In particular, exposure to trace amounts of antibiotics at early life can result in the alteration of microbiota and metabolic networks, which further accelerate the possible development of resistant strains [8,9]. Previous studies in mice have shown that subtherapeutic concentrations of penicillin exposure at early life interfered with the development of the intestinal immune system [10] and induced metabolic changes due to altered intestinal microbiota [11].

Our recent study has shown that trace amounts of antibiotic in feed exacerbated the detrimental effects of enterotoxigenic $E$. coli (ETEC) infection by increasing diarrhea and systemic inflammation in weanling pigs [12]. The exact mechanisms for the exacerbation of ETEC infection by trace amounts of antibiotic has not been determined, but it has been suggested that trace concentrations of antibiotics can act as signaling molecules to trigger specific bacterial responses [13]. Therefore, the objective of this study was 
to explore the impacts of feeding trace levels of antibiotic on host metabolic profiles and colon microbiota of weaned pigs experimentally infected with ETEC F18.

\section{Materials And Methods}

\section{Animals, Housing, Experimental Design, and Diet}

The protocol for this study was reviewed and approved by the Institutional Animal Care and Use Committee at the University of California, Davis (IACUC \#19322). Samples analyzed here were obtained as described in a previously published study by Kim et al. [12]. Briefly, 34 weanling pigs (crossbred; initial BW: $6.88 \pm 1.03 \mathrm{~kg}$ ) with an equal number of gilts and barrows were randomly assigned to one of three treatments in a randomized complete block design with body weight within sex and litter as the blocks and pig as the experimental unit. The 3 dietary treatments included: 1 ) the complex nursery basal diet (control; CON), 2) addition of $0.5 \mathrm{mg} / \mathrm{kg}$ carbadox (trace amounts of antibiotic; TRA) to the basal diet, or 3) addition of $50 \mathrm{mg} / \mathrm{kg}$ carbadox (label-recommended dose of antibiotic; REC) to the basal diet. All diets were formulated to meet pig nutritional requirements and provided as mash form throughout the experiment.

After 7 days of adaptation, all pigs were orally inoculated with $3 \mathrm{~mL}$ of $E$. coli $\mathrm{F} 18$ using a disposable Luer-lock syringe for 3 consecutive days from d 0 post-inoculation (PI). The E. coli $\mathrm{F} 18$ was originally isolated from a field disease outbreak by the University of Illinois Veterinary Diagnostic Lab (isolate number: U.IL-VDL \# 05-27,242) and expresses heat-labile toxin (LT), heat-stable toxin b (STb), and Shigalike toxin (SLT-2). The E. coli F18 inoculums were provided at $10^{10}$ colony-forming unit (CFU) per $3 \mathrm{~mL}$ dose in phosphate-buffered saline (PBS). This dose caused mild diarrhea in the current study as reported in Kim et al. [12], which is consistent with our previous published researches [14-16]. Growth performance, blood profiles, and immune responses were also reported in previous work [12].

\section{Sample Collections}

Sixteen pigs ( 6 pigs in CON, 4 pigs in TRA, and 6 pigs in REC) were randomly selected and euthanized on d $5 \mathrm{PI}$ near the peak of infection, and the remaining pigs ( 6 pigs in CON, 5 pigs in TRA, and 7 pigs in REC) were euthanized at the end of the experiment $(\mathrm{d} 11 \mathrm{PI})$ that was the recovery period of the infection. The selection of necropsy time was based on the results of clinical observations and immune response parameters that were reported in previously published research using the same $E$. colistrain and inoculation dose $[15,16]$. Before euthanasia, pigs were anesthetized with a 1-mL mixture of $100 \mathrm{mg}$ telazol, $50 \mathrm{mg}$ ketamine, and $50 \mathrm{mg}$ xylazine (2:1:1) by intramuscular injection. After anesthesia, intracardiac injection with 78 mg sodium pentobarbital (Vortech Pharmaceuticals, Ltd., Dearborn, MI) per $1 \mathrm{~kg}$ of BW was used to euthanize each pig. Blood samples were collected from the jugular vein of all pigs without EDTA to yield serum before $E$. coli challenge (d 0), and on d 5, and $11 \mathrm{PI}$. Serum samples were collected by centrifuging approximately $5 \mathrm{~mL}$ of whole blood samples at $20^{\circ} \mathrm{C}$ at $1500 \times \mathrm{g}$ for 15 
min and immediately stored at $-80^{\circ} \mathrm{C}$ until untargeted metabolomics analysis. Colon digesta were collected from the distal colon of pigs on $\mathrm{d} 5$ and $11 \mathrm{PI}$ and immediately snap-frozen in liquid nitrogen and stored at $-80^{\circ} \mathrm{C}$ for untargeted metabolomics and microbiome analysis. Untargeted Metabolomics

\section{Analysis}

The untargeted metabolomics analysis was performed by the NIH West Coast Metabolomics Center using gas chromatography (Agilent 6890 gas chromatograph controlled using Leco ChromaTOF software version 2.32) coupled with time-of-flight mass spectrometry (GC/TOF-MS) (Leco Pegasus IV time-of-flight mass spectrometer controlled using Leco ChromaTOF software version 2.32). Metabolite extraction was performed following procedures described previously [17]. Briefly, frozen colon digesta samples (approximately $10 \mathrm{mg}$ ) were homogenized using a Retsch ball mill for $30 \mathrm{~s}$ at 25 times/s. After homogenization, a prechilled $\left(-20^{\circ} \mathrm{C}\right)$ extraction solution (isopropanol/acetonitrile/water at the volume ratio $3: 3: 2$, degassed with liquid nitrogen) was added at a volume of $1 \mathrm{~mL} / 20 \mathrm{mg}$ of sample. Samples were then vortexed and shaken for metabolite extraction. After centrifugation at $12,800 \times \mathrm{g}$ for $2 \mathrm{~min}$, the supernatant was collected and divided into two equal aliquots and concentrated at room temperature for $4 \mathrm{~h}$ in a cold-trap vacuum concentrator (Labconco Centrivap, Kansas City, MO, USA). To separate complex lipids and waxes, the residue was re-suspended in $500 \mu \mathrm{L}$ of $50 \%$ aqueous acetonitrile and centrifuged at $12,800 \times \mathrm{g}$ for $2 \mathrm{~min}$. The resultant supernatant was collected and concentrated in the vacuum concentrator. Dried sample extracts were derivatized and mixed with internal retention index markers (fatty acid methyl esters with the chain length of C8 to C30). The samples were injected for GC/TOF analysis, and all samples were analyzed in a single batch [17].

\section{Gut Microbiota In Distal Colon}

Bacterial DNA was extracted from digesta samples using the Quick-DNA Fecal/Soil Microbe Kit (Zymo Research, Irvine, CA, USA) following the manufacturer's instructions. Extracted bacterial DNA was amplified with PCR, targeting the $V 4$ region of the $16 \mathrm{~S}$ rRNA gene with primers $515 \mathrm{~F}$ (5'XXXXXXXXGTGTGCCAGCMGCCGCGGTAA-3') with an 8 bp barcode (X) and Illumina adapter (GT) and 806 R (5'-GGACTACHVGGGTWTCTAAT-3') [18]. Amplification included thermocycling conditions of $94^{\circ} \mathrm{C}$ for $3 \mathrm{~min}$ for denaturation, 35 cycles of $94^{\circ} \mathrm{C}$ for $45 \mathrm{~s}, 50^{\circ} \mathrm{C}$ for $1 \mathrm{~min}, 72^{\circ} \mathrm{C}$ for $1.5 \mathrm{~min}$, and $72^{\circ} \mathrm{C}$ for 10 min (final elongation). To reduce PCR bias, each sample was amplified in triplicate. Each PCR reaction included $2 \mu \mathrm{L}$ of template DNA, $0.5 \mu \mathrm{L}(10 \mu \mathrm{mol} / \mathrm{L})$ of barcoded forward primer, $0.5 \mu \mathrm{L}(10 \mu \mathrm{mol} / \mathrm{L})$ of reverse primer, $12.5 \mu \mathrm{L}$ of GoTaq $2 X$ Green Master Mix (Promega, Madison, WI, USA), and 9.5 $\mu \mathrm{L}$ of nuclease-free water. The triplicate PCR products were pooled and subjectively quantified based on the brightness of the bands on a $2 \%$ agarose gel with SYBR safe (Invitrogen Co., Carlsbad, CA, USA). All amplicons were then pooled at equal amounts and further purified using the QIAquick PCR Purification Kit (Qiagen, Hilden, Germany). The purified library was submitted to the UC Davis Genome Center DNA Technologies Core for 250 bp paired-end sequencing on the Illumina MiSeq platform (Illumina, Inc. San Diego, CA, USA). 
The software sabre (https://github.com/najoshi/sabre) was used to demultiplex and remove barcodes from raw sequences. Sequences were then imported into Quantitative Insights Into Microbial Ecology 2 (QIIME2; version 2018.6) for downstream filtering and bioinformatics analysis [19, 20]. Plugin q2-dada2 [21] was used for quality control and constructing features. Taxonomic classification was assigned using the feature-classifier plugin trained with the SILVA rRNA database $99 \%$ Operational Taxonomic Units (OTU), version $132[22,23]$.

\section{Data Analysis}

The metabolomics data were analyzed using different modules of a web-based platform, MetaboAnalyst 5.0 (https://www.metaboanalyst.ca) [24]. Data were filtered for peaks with detection rates less than $30 \%$ of missing abundances and normalized using logarithmic transformation and auto-scaling. Mass univariate analysis was performed using one-way ANOVA followed by Tukey's post hoc test (adjusted $P \leq$ 0.05). Fold change analysis and t-tests were also conducted to determine the fold change and significance of each identified metabolite. Statistical significance was declared at a false discovery rate (FDR, Benjamini and Hochberg correction; q) $q<0.2$ and fold change $>2.0$. Partial least squares discriminant analysis (PLS-DA) was carried out to further identify discriminative variables (metabolites) among the treatment groups. Pathway analysis and metabolite set enrichment analysis were performed on identified metabolites that had a Variable Importance in Projection (VIP) score $>1$.

Data visualization and statistical analysis for colon microbiota were conducted using R (version 3.6.1). Two alpha diversity indices, Chao 1 and Shannon, were calculated using the phyloseq package. Relative abundance was calculated using the phyloseq package and visualized using the ggplot2 package in R. Relative abundance data were aggregated at various taxonomical levels. Shapiro-Wilk normality test and Bartlett test were used to verify normality and constant variance, respectively, in alpha diversity and relative abundance. Shannon index was analyzed using ANOVA with the statistical model, including sample collection days within treatment as fixed effects. Significance in Chao1 index and relative abundance was observed using Kruskal-Wallis rank-sum test followed by a Conover test for multiple pairwise comparisons using the agricolae package. Beta diversity was calculated based on the BrayCurtis dissimilarity for principal coordinates analysis (PCOA). The homogeneity of multivariate dispersions was tested by the vegan package using the betadisper function, before the adonis function was used to calculate PERMANOVA with 999 replicate permutations. Statistical significance and tendency were considered at $P<0.05$ and $0.05 \leq P<0.10$, respectively.

\section{Results}

\section{Metabolite Profiles in Serum}

A total of 354 (134 identified and 220 unidentified) metabolites were detected in serum samples. Based on VIP scores and relative abundance, 3 metabolites (fructose, mannonic acid, and propyleneglycol) were up-regulated by TRA, compared with the pigs in REC on d 0 before $E$. coli challenge (Table 1). 
Supplementation of REC changed the abundances of 6 metabolites ( 2 up-regulated and 4 downregulated) compared with CON, while REC changed 16 metabolites (6 up-regulated and 10 downregulated) in comparison with TRA on d $5 \mathrm{PI}$. On d $11 \mathrm{PI}$, chenodeoxycholic acid was enriched, while glycerol and inositol-4-monophosphate were reduced in the CON group compared with REC. Pigs in TRA had greater chenodeoxycholic acid than pigs in REC, but 5 metabolites ( $p$-tolyl glucuronide, glycerol, mannitol, 2-ketoisocaproic acid, and inositol-4-monophosphate) were decreased in pigs supplemented with TRA compared with pigs in REC. No differential metabolites were identified when comparing CON vs. TRA throughout the experiment. Based on the identified metabolites, a PLS-DA score plot with 95\% confidence ranges (shaded areas) showed a clear separation between the TRA and REC groups throughout the experiment (Figure 1). To further explore the metabolic profile differences among two dietary treatments, PLS-DA was performed for the following comparisons: (1) TRA vs. REC, and (2) CON vs. REC on $\mathrm{d} 0$ before $E$. coli challenge, $\mathrm{d} 5$ and $\mathrm{d} 11 \mathrm{PI}$. The score plot again distinguished the TRA from the REC, and also revealed the metabolic profile differences between CON and REC (Figure S1).

Pathway analysis and metabolite set enrichment analysis were performed on metabolites in serum with $\mathrm{VIP}>1$. On $\mathrm{d} 0$ before $E$. coli challenge, inositol phosphate metabolism, glyoxylate and discarboxylate metabolism, glycine, serine and threonine metabolism, citrate cycle, and ascorbate and aldarate metabolism were the most affected metabolic pathways when comparing CON with REC (Figure S2A, C). Citrate (TCA) cycle, arginine biosynthesis, and alanine, aspartate, and glutamate metabolism were the most affected metabolic pathways when TRA was compared with REC (Figure S2B, D). On d 5 PI, aminoacyl-tRNA biosynthesis, glycine, serine, and threonine metabolism, and phenylalanine, tyrosine, and tryptophan biosynthesis were the most affected metabolic pathways when comparing CON vs. REC (Figure 2A, C), while aminoacyl-tRNA biosynthesis, alanine, aspartate, and glutamate metabolism, and glycolysis and gluconeogenesis were the most affected metabolic pathways in a comparison of TRA vs. REC (Figure 2B, D). On d $11 \mathrm{PI}$, arginine biosynthesis, alanine, aspartate and glutamate metabolism, Dglutamine and D-glutamate metabolism, pyrimidine metabolism, and citrate cycle were the most affected metabolic pathways when comparing CON with REC (Figure S3A, C). Arginine biosynthesis, aminoacyltRNA biosynthesis, alanine, aspartate, and glutamate metabolism, and D-glutamine and D-glutamate metabolism were the most affected metabolic pathways in a comparison of TRA with REC (Figure S3B, D).

\section{Metabolite Profiles in Distal Colon Digesta}

A total of 398 (178 identified and 220 unidentified) metabolites were detected in colon digesta. Based on VIP score and relative abundance, 12 metabolites ( 9 up-regulated and 3 down-regulated) were differentiated on d $5 \mathrm{PI}$, and one metabolite, inosine, was decreased on d $11 \mathrm{PI}$ in pigs fed with TRA when compared with pigs in the REC group (Table 2). No differential metabolites were identified when comparing CON vs. TRA, and CON vs. REC at $d 5$ and $11 \mathrm{PI}$. Based on the identified metabolites, a PLS-DA score plot with 95\% confidence ranges (shaded areas) showed a clear separation between the TRA and REC groups at both PI time points (Figure 3 ). The PLS-DA score plots in a pairwise manner also clearly separated TRA from REC on d 5 and 11 PI (Figure S4). 
Pathway analysis and metabolite set enrichment analysis were performed on metabolites in colon digesta with VIP $>1$. Starch and sucrose metabolism, purine metabolism, arginine biosynthesis, and arginine and proline metabolism were the most affected metabolic pathways when TRA group was compared with the REC group on d $5 \mathrm{PI}$ (Figure 4A, C). Aminoacyl-tRNA biosynthesis, arginine biosynthesis, pentose and glucuronate interconversions, arginine and proline metabolism, alanine, aspartate, and glutamate metabolism, glutathione metabolism, and glyoxylate and dicarboxylate metabolism were the most affected metabolic pathways on d 11 PI when TRA group was compared with the REC group (Figure 4B, D).

\section{Microbial Profiles in Distal Colon Digesta}

A total of 481,102 qualified reads were obtained with a mean of 15,034 reads per sample. A total of 3,561 OTUs were identified in the current experiment. No differences were observed in the alpha diversity of distal colon content among dietary treatments on d 5 and d 11 PI. Both Chao 1 and Shannon indices of the distal colon content were lower $(P<0.05)$ on $\mathrm{d} 11 \mathrm{PI}$ than $\mathrm{d} 5 \mathrm{PI}$ for pigs in the CON group (Figure S5). Beta diversity (Adonis analysis based on the Bray-Curtis distance) indicated that day (days $\mathrm{PI}$ ) was a significant factor associated with composition distance $\left(R^{2}=0.11, P<0.05\right.$; Figure S6). Compositional differences of the distal colon microbiota were also observed between CON vs. REC and TRA vs. REC on d 5 and $\mathrm{d} 11 \mathrm{PI}$ (Pairwise-Adonis, $P<0.05$; Figure 66 ).

The dominant phyla in distal colon content were Firmicutes, Bacteroidetes, Proteobacteria, and Actinobacteria, regardless of treatment or sampling day (Figure S7). Pigs in the TRA or REC group had a lower $(P<0.05)$ relative abundance of Actinobacteria than pigs in the CON group on $\mathrm{d} 11 \mathrm{PI}$. Within the Firmicutes phylum (Figure 5), pigs in the TRA group had lower $(P<0.05)$ relative abundance of Lactobacillaceae (8.91\% vs. $21.33 \%$ ) than pigs in REC on d $5 \mathrm{Pl}$, whereas REC had lower $(P<0.05)$ relative abundance of Lactobacillaceae $(5.82 \%$ vs. $23.90 \%$ or $27.69 \%)$ than pigs in the CON or TRA groups on $\mathrm{d} 11 \mathrm{PI}$. Pigs in the REC group had higher $(P<0.05)$ relative abundance of Clostridiaceae (17.14\% vs. $1.45 \%)$ and Streptococcaceae $(10.09 \%$ vs. $0.21 \%)$, but lower $(P<0.05)$ relative abundance of Lachnospiraceae (20.25\% vs. $27.44 \%$ ) in the distal colon on $\mathrm{d} 11 \mathrm{PI}$ than on $\mathrm{d} 5 \mathrm{PI}$. Within the Bacteroidetes phylum (Figure 6), pigs in the TRA group had reduced $(P<0.05)$ relative abundance of Muribaculaceae ( $0.60 \%$ vs. $2.46 \%$ ) and Rikenellaceae ( $0.61 \%$ vs. $3.09 \%)$ in distal colon on $\mathrm{d} 11 \mathrm{PI}$ than on d $5 \mathrm{PI}$. On d $11 \mathrm{PI}$, pigs in the CON group had higher $(P<0.05)$ relative abundance of Prevotellaceae $(13.78 \%$ vs. $9.32 \%)$ in distal colon content, compared with pigs in the REC group.

\section{Discussion}

In-feed antibiotics can influence nutrient metabolism and many biological processes in pigs by altering microbiota and metabolites $[25,26]$. However, little is known about the impacts of trace amounts of antibiotics on metabolic and microbial changes in piglets, especially under disease-challenged conditions. The present study investigated the alteration of metabolic pathways in the serum and colon digesta by using an untargeted metabolomics approach when pigs were supplemented with different 
levels of the antibiotic carbadox. Results from the current study highlighted that supplementing labelrecommended doses of antibiotics altered metabolomic markers related to nutrient metabolism in the serum and colon digesta. Moreover, supplementation of different levels of antibiotic modified microbial community composition and diversity to a different extent in the colon digesta of pigs challenged with ETEC F18. Our previous research reported that supplementing the label-recommended dose of antibiotic enhanced disease resistance and promoted growth, whereas supplementing trace amounts of antibiotic exacerbated the detrimental effects of ETEC F18 infection on performance and diarrhea, and systemic inflammation of weaned pigs [12]. Results from the current study will help us to understand the negative impacts of trace amounts of antibiotic on performance and health of young pigs by focusing on the gut microbiome and their metabolites and the host metabolism.

The metabolomics approach exploits high-throughput analytical measurements to identify host and microbiota metabolites and associated biological changes that are affected by internal or external factors to maintain homeostasis [27]. In the present study, differences in the metabolic profiles of serum and colon digesta were found predominately between pigs supplemented with trace amounts of antibiotic and label-recommended dose of antibiotic, especially during the peak infection period (d $5 \mathrm{PI})$. These findings suggest the comparative dose-response metabolic effects of antibiotics during ETEC infection in weaned pigs.

In-feed antibiotics mediate growth enhancement as a result of improved nutrient utilization in pigs. Growing evidence suggests that the administration of in-feed antibiotics can enhance nutrient digestibility and regulate the nutrient metabolism of the host [28]. The bacteriostatic activity of in-feed antibiotics may also impact the intestinal microbial metabolites by reducing growth depressing microbiota [29]. It was reported that in-feed antibiotics at a subtherapeutic concentration could enhance amino acid availability in piglets, as indicated by increased serum metabolomic markers that are associated with amino acid metabolism [25]. Amino acid metabolism is extremely important to support animal growth, maintain homeostasis, and regulate other biological processes in the host and intestinal microbiota [30,31]. In the present study, metabolites related to amino acid metabolism (2-hydroxyvaleric acid, pipecolinic acid, histidine, and creatine) were enriched in the serum of pigs fed with the labelrecommended antibiotic dose compared with pigs in the trace amounts of antibiotic group. This was likely due to the reduced peptide catabolism initiated by microbial protease activities when feeding labelrecommended dose of antibiotic [32]. However, 2-hydroxyvaleric acid, a metabolomic marker of branchedchain amino acid catabolism, was observed to be reduced in the colon digesta of pigs fed with labelrecommended dose antibiotic compared with the trace amounts of antibiotic group. These observations are in agreement with a previous research, in which Mu et al. [25] also reported that increased serum metabolites related to amino acid metabolism were concomitant with a decrease in jejunal metabolites associated with amino acid metabolism in pigs fed with a mixture of antibiotics at a growth-promoting dose. Thus, these results suggest that the systemic interplay between microbiota and metabolite profiles was promoted by feeding label-recommended dose of antibiotics. A previous study using metagenomic analysis also observed that antibiotics at subtherapeutic doses reduced the abundance of clusters of orthologous groups involved in protein metabolism in the fecal microbiota of pigs [33]. Consistent with 
performance data and clinical signs [12], it is not surprising to observe that trace amount of antibiotics had different impacts on serum and colon digesta metabolites that are associated with amino acid metabolism when compared with label-recommended dose of antibiotic. Previous in vitro research suggested that $E$. coli cells stimulated cellular functions and metabolic modifications of amino acid catabolism upon exposure to the antibiotic ampicillin at below the minimal inhibitory concentrations (sub-MIC) [34]. More specifically, E. coli cells treated with sub-MIC ampicillin resulted in increased amino acid depletion in Luria-Bertani (LB) media due to stress responses, which provided amino acids as a major energy source for cultured cells. This finding indicates that the alteration of metabolomic markers of amino acid metabolism caused by trace amounts of antibiotic in the current study may be related to the depletion of amino acids during the host response to ETEC infection. Subsequently, less amounts of amino acids might be available to support the growth of the pigs when they were challenged with ETEC and supplemented with trace amount of antibiotics.

Carbohydrate metabolism is essential to support the virulence of pathogenic enterobacteria [35]. It has been reported that the colonization of pathogenic $E$. coli in the mouse intestine was supported by the catabolism of several carbohydrates, including galactose, fucose, mannose, and maltose [36, 37]. In the present study, metabolomic markers related to galactose metabolism (glycerol and myo-inositol) and carbohydrate digestion and absorption (maltotriose) were enriched in the serum or colon digesta from pigs supplemented with trace amounts of antibiotic. This finding suggests that trace amounts of antibiotic may assist in constitution of an ecological niche for ETEC F18 colonization in the intestine of pigs, rather than exhibit its antibacterial activity. Besides the carbohydrate utilization by pathogens to colonize, carbohydrate metabolism is also vital for the systemic inflammatory response [38]. Baurhoo et al. [39] reported that a significant mobilization and catabolism of carbohydrates were observed in the intestine of chickens during LPS-induced systemic inflammation. In the present animal study, trace amounts of antibiotic exacerbated the intestinal and systemic inflammatory status of ETEC F18 challenged pigs [12]. Thus, the increased metabolites associated with carbohydrate metabolism in pigs supplemented with trace amount of antibiotics during the peak of ETEC infection indicates that these pigs may utilize more carbohydrates as energy sources to support their immune responses and recovery processes against ETEC F18 instead of growth.

Interestingly, supplementation of trace amounts of antibiotic also enriched serum metabolomic markers of purine metabolism (hypoxanthine and guanine) during the peak of ETEC infection. A previous in vitro study reported that Pasteurella multocida significantly increased the expression of proteins involved in purine synthesis and metabolism, in response to sub-MIC antibiotics, including amoxicillin, chlortetracycline, and enrofloxacin [40]. $\mathrm{Ng}$ et al. [41] also demonstrated that extremely low concentrations of antibiotics, such as tetracycline and macrolide, upregulated the expression of genes associated with purine metabolism in Streptococcus pneumoniae. The metabolites involved in purine metabolism are often upregulated in the activated immune cells as important immune signaling molecules [42]. For instance, previous research reported that mice infected with $E$. coli had enriched plasma metabolites that are linked to the purine metabolic pathway [43]. Likewise, growing evidence also suggests that trace concentrations of antibiotics may perform as signaling agents and trigger special 
bacterial responses, such as increased purine metabolism, following an infection [42, 44], Thus, our results indicate that purine metabolism might contribute to the elevated systemic inflammation in pigs fed with trace amounts of antibiotic [12].

The composition and diversity of gut microbial communities in pigs are greatly impacted by their age, health status, and nutrient components in feed [45-47]. To test the impacts of trace amounts of antibiotic on gut microbiota diversity, distal colon contents were collected, and 16S rRNA gene sequencing was performed. Consistent with previously published research, antibiotics-treated at recommended concentrations clustered separately from non-treated groups [33,48], indicating that antibiotics administration at label-recommended dose altered colon microbiota composition and diversity. However, there was no clear separation in distal colon microbiota between pigs supplemented with trace amounts of antibiotic and pigs in the control group.

The three most abundant phyla found in the colon digesta of pigs in the present study were Firmicutes, Bacteroidetes, and Proteobacteria, which was consistent with previously published research $[49,50]$. Within the Firmicutes and Bacteroidetes phyla, the relative abundance of Lachnospiraceae and Lactobacillaceae were enriched in the distal colon of pigs supplemented with label-recommended dose of antibiotic, but the relative abundance of Lactobacillaceae was reduced in colon digesta of trace amounts of antibiotic pigs during the peak infection period. Lachnospiraceae are capable of producing butyric acid, which provides energy for other microbes and host epithelial cells and prevents the growth of other microbes in the digestive tract [51,52]. Moreover, Lactobacillaceae were reported to be positively correlated with feed efficiency [53] and nitrogen, energy, cellulose, and hemicellulose digestibility in pigs [54]. Although the exact mechanism of antimicrobial effects is not yet clear, Lactobacillaceae are known for their health-promoting effects and for their ability to inhibit intestinal pathogens such as $E$. coli and Salmonella [55]. Thus, Lachnospiraceae and Lactobacillaceae have been proposed and investigated as biomarkers to predict the health status of pigs $[56,57]$. Rhouma et al. [58] demonstrated that the ETEC F4 challenge suppressed the relative abundance of Lachnospiraceae and Lactobacillaceae in fecal contents of pigs, compared with unchallenged pigs. In addition, Duo et al. [59] also reported that diarrheic pigs, in natural post-weaning diarrhea, had a lower abundance of Lachnospiraceae and Lactobacillaceae in feces, compared with healthy pigs. Therefore, the modified intestinal microbial environment, including the enhanced presence of Lachnospiraceae and Lactobacillaceae, may contribute to the accelerated recovery from ETEC 18 infection in pigs supplemented with label-recommended dose of antibiotics.

Previous studies have also reported the contribution of intestinal microbiota to weight gain in pigs. For example, Kim et al. [60] observed that Clostridiaceae in the distal gut of pigs were positively correlated with weight gain, while Unno et al. [61] reported a negative correlation between weight and Prevotellaceae in feces when pigs were supplemented with different types of antibiotics. In the present study, pigs fed label-recommended dose antibiotic had increased relative abundance of Clostridiaceae but reduced relative abundance of Prevotellaceae compared to pigs in the control group on $\mathrm{d} 11 \mathrm{PI}$. These observations are consistent with the literature and confirmed the effectiveness of label-recommended dose of antibiotic for growth-promoting purposes [12]. 
In conclusion, the metabolomics and microbiome approaches in the present study identified the differential metabolites and their pathways in the serum and distal colon digesta of ETEC F18 challenged pigs. Compared with label-recommended dose of antibiotic, trace amounts of antibiotic oppositely affected metabolomic markers related to the metabolisms of amino acids, carbohydrates, and purine. Pigs administered label-recommended dose of antibiotic had marked modulation of microbial composition, which may be highly correlated with their enhanced growth performance and disease resistance in weaned pigs. The impacted metabolic pathways and colonic microbial shift may also be closely associated with the slow growth and delayed recovery from ETEC infection of weaned pigs supplemented with trace amounts of antibiotic. Future studies will consider incorporating targeted metabolomics and metagenomics to provide more insights into the potential risk of trace amounts of antibiotic on the host response to ETEC infection. The exploration of metabolomic markers and gut microbiome interaction will be important to decipher the mechanisms of how trace amounts of antibiotic negatively impact the health of young animals.

\section{Abbreviations}

FDR

False discovery rate

ETEC

Enterotoxigenic E. coli

MIC

Minimal inhibitory concentrations

OTU

Operational taxonomic units

$\mathrm{PI}$

Post-inoculation

PLS-DA

Partial least squares discriminant analysis

PCoA

Principal coordinates analysis

VIP

Variable importance in the projection.

\section{Declarations}

\section{Ethics approval}

The protocol for this study was reviewed and approved by the Institutional Animal Care and Use Committee at the University of California, Davis (IACAC \#19322). The study was conducted at the Cole Facility at the University of California, Davis. 


\section{Consent for publication}

Not applicable.

\section{Availability of data and materials}

All data generated or analyzed during this study are available from the corresponding author upon reasonable request.

\section{Competing interests}

The authors declare that they have no competing interests.

\section{Funding}

This project was supported by the United States Department of Agriculture (USDA) National Institute of Food and Agriculture (NIFA), multistate projects W4002 and NC1202.

\section{Author's contributions}

The contributions of the authors were as follows: K. K. conducted the experiment and wrote the manuscript. C. J. and P. J. helped to conduct animal trial, part of the laboratory work, data analysis, and helped to revise the manuscript. Y. L. was the principal investigator. Y. L. designed the experiment, oversaw the development of the study and wrote the last version of the manuscript. The authors declare no conflicts of interest. The authors read and approved the final manuscript.

\section{Acknowledgments}

Not applicable.

\section{Conflict of interest statement}

The authors disclose that there was no conflict of interest.

\section{References}

1. Ben Y, Fu C, Hu M, Liu L, Wong MH, Zheng C. Human health risk assessment of antibiotic resistance associated with antibiotic residues in the environment: A review. Environ Res. 2019;169:483-493. doi:10.1016/j.envres.2018.11.040.

2. Jechalke S, Heuer H, Siemens J, Amelung W, Smalla K. Fate and effects of veterinary antibiotics in soil. Trends Microbiol. 2014;22:536-545. doi:10.1016/j.tim.2014.05.005.

3. Wei R, Ge F, Huang S, Chen M, Wang R. Occurrence of veterinary antibiotics in animal wastewater and surface water around farms in Jiangsu Province, China. Chemosphere. 2011;82:1408-1414. doi:10.1016/j.chemosphere.2010.11.067. 
4. Hamscher G, Hartung J. Veterinary antibiotics in dust: sources, environmental concentrations, and possible health hazards. Pharm Environ. Springer; 2008. p. 95-102.

5. Manzetti S, Ghisi R. The environmental release and fate of antibiotics. Mar Pollut Bull. 2014;79:7-15. doi:10.1016/j.marpolbul.2014.01.005.

6. Sarmah AK, Meyer MT, Boxall ABA. A global perspective on the use, sales, exposure pathways, occurrence, fate and effects of veterinary antibiotics (VAs) in the environment. Chemosphere. 2006;65:725-759. doi:10.1016/j.chemosphere.2006.03.026.

7. Chen J, Ying G-G, Deng W-J. Antibiotic residues in food: extraction, analysis, and human health concerns. J Agric Food Chem. 2019;67:7569-7586. doi:10.1021/acs.jafc.9b01334.

8. Nisha AR. Antibiotic residues-A global health hazard. Vet. World. 1:375-377. Available from: https://agris.fao.org/agris-search/search.do?recordID=DJ2012059763.

9. Davies J, Spiegelman GB, Yim G. The world of subinhibitory antibiotic concentrations. Curr Opin Microbiol. 2006;9:445-453. doi:10.1016/j.mib.2006.08.006.

10. Jin S, Zhao D, Cai C, Song D, Shen J, Xu A, et al. Low-dose penicillin exposure in early life decreases Th17 and the susceptibility to DSS colitis in mice through gut microbiota modification. Sci Rep. 2017;7:43662. doi:10.1038/srep43662.

11. Cox LM, Yamanishi S, Sohn J, Alekseyenko A V, Leung JM, Cho I, et al. Altering the intestinal microbiota during a critical developmental window has lasting metabolic consequences. Cell. 2014;158:705-721. doi:10.1016/j.cell.2014.05.052.

12. Kim K, He Y, Jinno C, Kovanda L, Li X, Song M, et al. Trace amounts of antibiotic exacerbated diarrhea and systemic inflammation of weaned pigs infected with a pathogenic Escherichia coli. J Anim Sci. 2021;99:1-13. doi:10.1093/jas/skab073.

13. Fajardo A, Martínez JL. Antibiotics as signals that trigger specific bacterial responses. Curr Opin Microbiol. 2008;11:161-167. doi:10.1016/j.mib.2008.02.006.

14. Liu Y, Song M, Che TM, Almeida JAS, Lee JJ, Bravo D, et al. Dietary plant extracts alleviate diarrhea and alter immune responses of weaned pigs experimentally infected with a pathogenic Escherichia coli. J Anim Sci. 2013;91:5294-5306. doi:10.2527/jas.2012-6194.

15. Kim K, Ehrlich A, Perng V, Chase JA, Raybould H, Li X, et al. Algae-derived $\beta$-glucan enhanced gut health and immune responses of weaned pigs experimentally infected with a pathogenic $E$. coli. Anim Feed Sci Technol. 2019;248:114-125. doi:10.1016/j.anifeedsci.2018.12.004.

16. Kim K, He Y, Xiong X, Ehrlich A, Li X, Raybould H, et al. Dietary supplementation of Bacillus subtilis influenced intestinal health of weaned pigs experimentally infected with a pathogenic $E$. coli. J Anim Sci Biotechnol. 2019;10:52-64. doi:10.1186/s40104-019-0364-3.

17. Fiehn O, Wohlgemuth G, Scholz M, Kind T, Lee DY, Lu Y, et al. Quality control for plant metabolomics: reporting MSI-compliant studies. Plant J. 2008;53:691-704. doi: 10.1111/j.1365-313X.2007.03387.x.

18. Caporaso JG, Lauber CL, Walters WA, Berg-Lyons D, Huntley J, Fierer N, et al. Ultra-high-throughput microbial community analysis on the Illumina HiSeq and MiSeq platforms. ISME J. 2012;6:16211624. doi:10.1038/ismej.2012.8. 
19. Bolyen E, Rideout JR, Dillon MR, Bokulich NA, Abnet CC, Al-Ghalith GA, et al. Reproducible, interactive, scalable and extensible microbiome data science using QIIME 2. Nat Biotechnol. 2019;37:852-857. doi:10.1038/s41587-019-0209-9.

20. Caporaso JG, Kuczynski J, Stombaugh J, Bittinger K, Bushman FD, Costello EK, et al. QIIME allows analysis of high-throughput community sequencing data. Nat Methods. 2010;7:335-336. doi:10.1038/nmeth.f.303.

21. Callahan BJ, McMurdie PJ, Rosen MJ, Han AW, Johnson AJA, Holmes SP. DADA2: high-resolution sample inference from Illumina amplicon data. Nat Methods. 2016;13:581-583. doi:10.1038/nmeth.3869.

22. Quast C, Pruesse E, Yilmaz P, Gerken J, Schweer T, Yarza P, et al. The SILVA ribosomal RNA gene database project: improved data processing and web-based tools. Nucleic Acids Res. 2012;41:D590596. doi:10.1093/nar/gks1219.

23. Bokulich NA, Kaehler BD, Rideout JR, Dillon M, Bolyen E, Knight R, et al. Optimizing taxonomic classification of marker-gene amplicon sequences with QIIME 2's q2-feature-classifier plugin. Microbiome. 2018;6:1-17. doi:10.1186/s40168-018-0470-z.

24. Pang Z, Chong J, Zhou G, de Lima Morais DA, Chang L, Barrette M, et al. MetaboAnalyst 5.0: narrowing the gap between raw spectra and functional insights. Nucleic Acids Res. 2021;49:W38896. doi: 10.1093/nar/gkab382.

25. Mu C, Yang Y, Yu K, Yu M, Zhang C, Su Y, et al. Alteration of metabolomic markers of amino-acid metabolism in piglets with in-feed antibiotics. Amino Acids. 2017;49:771-781. doi:10.1007/s00726017-2379-4.

26. Pi Y, Gao K, Peng Y, Mu CL, Zhu WY. Antibiotic-induced alterations of the gut microbiota and microbial fermentation in protein parallel the changes in host nitrogen metabolism of growing pigs. Animal. 2019;13:262-272. doi:10.1017/S1751731118001416.

27. Weckwerth W. Metabolomics in systems biology. Annu Rev Plant Biol. 2003;54:669-689. doi:10.1146/annurev.arplant.54.031902.135014.

28. Gaskins HR, Collier CT, Anderson DB. Antibiotics as growth promotants: mode of action. Anim Biotechnol. 2002;13:29-42. doi:10.1081/ABI0-120005768.

29. Brüssow H. Growth promotion and gut microbiota: insights from antibiotic use. Environ Microbiol. 2015;17:2216-2227. doi:10.1111/1462-2920.12786.

30. Christensen HN. Role of amino acid transport and countertransport in nutrition and metabolism. Physiol Rev. 1990;70:43-77. doi:10.1152/physrev.1990.70.1.43.

31. Wu G. Amino acids: metabolism, functions, and nutrition. Amino Acids. 2009;37:1-17. doi:10.1007/s00726-009-0269-0.

32. Romick-Rosendale LE, Goodpaster AM, Hanwright PJ, Patel NB, Wheeler ET, Chona DL, et al. NMRbased metabonomics analysis of mouse urine and fecal extracts following oral treatment with the broad-spectrum antibiotic enrofloxacin (Baytril). Magn Reson Chem. 2009;47:S36-46. doi:10.1002/mrc.2511. 
33. Looft T, Johnson TA, Allen HK, Bayles DO, Alt DP, Stedtfeld RD, et al. In-feed antibiotic effects on the swine intestinal microbiome. Proc Natl Acad Sci. 2012;109:1691-1696.

doi:10.1073/pnas.1120238109.

34. Mathieu A, Fleurier S, Frénoy A, Dairou J, Bredeche M-F, Sanchez-Vizuete P, et al. Discovery and function of a general core hormetic stress response in $E$. coli induced by sublethal concentrations of antibiotics. Cell Rep. 2016;17:46-57. doi:10.1016/j.celrep.2016.09.001.

35. Le Bouguénec $C$, Schouler C. Sugar metabolism, an additional virulence factor in enterobacteria. Int J Med Microbiol. 2011;301:1-6. doi:10.1016/j.ijmm.2010.04.021.

36. Fabich AJ, Jones SA, Chowdhury FZ, Cernosek A, Anderson A, Smalley D, et al. Comparison of carbon nutrition for pathogenic and commensal Escherichia coli strains in the mouse intestine. Infect Immun. 2008;76:1143-1152. doi:10.1128/IAI.01386-07.

37. Jones SA, Jorgensen M, Chowdhury FZ, Rodgers R, Hartline J, Leatham MP, et al. Glycogen and maltose utilization by Escherichia coli 0157:H7 in the mouse intestine. Infect Immun. 2008;76:25312540. doi:10.1128/IAl.00096-08.

38. Wolowczuk I, Verwaerde C, Viltart O, Delanoye A, Delacre M, Pot B, et al. Feeding our immune system: impact on metabolism. Clin Dev Immunol. 2008; 2008:639803. doi:10.1155/2008/639803.

39. Baurhoo B, Ferket P, Ashwell CM, de Oliviera J, Zhao X. Cell Walls of Saccharomyces cerevisiae differentially modulated innate immunity and glucose metabolism during late systemic inflammation. PLoS One. 2012;7:1-16. doi:10.1371/journal.pone.0030323.

40. Nanduri B, Lawrence ML, Peddinti DS, Burgess SC. Effects of subminimum inhibitory concentrations of antibiotics on the Pasteurella multocida proteome: a systems approach. Comp Funct Genomics. 2008;2008:254836. doi:10.1155/2008/254836.

41. Ng W-L, Kazmierczak KM, Robertson GT, Gilmour R, Winkler ME. Transcriptional regulation and signature patterns revealed by microarray analyses of Streptococcus pneumoniae R6 challenged with sublethal concentrations of translation inhibitors. Am Soc Microbiol. 2003;185:359-370. doi:10.1128/JB.185.1.359-370.2003.

42. Cekic C, Linden J. Purinergic regulation of the immune system. Nat Rev Immunol. 2016;16:177-192. doi:10.1038/nri.2016.4.

43. Yang JH, Bhargava P, McCloskey D, Mao N, Palsson BO, Collins JJ. Antibiotic-induced changes to the host metabolic environment inhibit drug efficacy and alter immune function. Cell Host Microbe. 2017;22:757-765.e3. doi: 10.1016/j.chom.2017.10.020.

44. Romero D, Traxler MF, López D, Kolter R. Antibiotics as signal molecules. Chem Rev. 2011;111:54925505. doi:10.1021/cr2000509.

45. Gresse R, Chaucheyras-Durand F, Fleury MA, Van de Wiele T, Forano E, Blanquet-Diot S. Gut microbiota dysbiosis in postweaning piglets: understanding the keys to health. Trends Microbiol. 2017;25:851-873. doi:10.1016/j.tim.2017.05.004.

46. Frese SA, Parker K, Calvert CC, Mills DA. Diet shapes the gut microbiome of pigs during nursing and weaning. Microbiome. 2015;3:28. doi:10.1186/s40168-015-0091-8. 
47. Guevarra RB, Hong SH, Cho JH, Kim BR, Shin J, Lee JH, et al. The dynamics of the piglet gut microbiome during the weaning transition in association with health and nutrition. J Anim Sci Biotechnol. 2018;9:54-63. doi:10.1186/s40104-018-0269-6.

48. Schokker D, Zhang J, Vastenhouw SA, Heilig HGHJ, Smidt H, Rebel JMJ, et al. Long-lasting effects of early-life antibiotic treatment and routine animal handling on gut microbiota composition and immune system in pigs. PLoS One. 2015;10:1-18. doi:10.1371/journal.pone.0116523.

49. Crespo-Piazuelo D, Estellé J, Revilla M, Criado-Mesas L, Ramayo-Caldas Y, Óvilo C, et al. Characterization of bacterial microbiota compositions along the intestinal tract in pigs and their interactions and functions. Sci Rep. 2018;8:12727. doi:10.1038/s41598-018-30932-6.

50. He Y, Jinno C, Kim K, Wu Z, Tan B, Li X, et al. Dietary Bacillus spp. enhanced growth and disease resistance of weaned pigs by modulating intestinal microbiota and systemic immunity. J Anim Sci Biotechnol. 2020;11:101-120. doi:10.1186/s40104-020-00498-3.

51. Meehan CJ, Beiko RG. A Phylogenomic view of ecological specialization in the Lachnospiraceae, a family of digestive tract-associated bacteria. Genome Biol Evol. 2014;6:703-713. doi:10.1093/gbe/evu050.

52. Guarner F, Malagelada J-R. Gut flora in health and disease. The Lancet. 2003;361:512-519. doi:10.1016/S0140-6736(03)12489-0.

53. Quan J, Cai G, Ye J, Yang M, Ding R, Wang X, et al. A global comparison of the microbiome compositions of three gut locations in commercial pigs with extreme feed conversion ratios. Sci Rep. 2018;8:4536-4546. doi:10.1038/s41598-018-22692-0.

54. Le Sciellour M, Labussière $E$, Zemb 0 , Renaudeau D. Effect of dietary fiber content on nutrient digestibility and fecal microbiota composition in growing-finishing pigs. PLoS One. 2018;13:e0206159. doi:10.1371/journal.pone.0206159.

55. Stecher B, Hardt W-D. The role of microbiota in infectious disease. Trends Microbiol. 2008;16:107114. doi:10.1016/j.tim.2007.12.008.

56. Guevarra RB, Lee JH, Lee SH, Seok M-J, Kim DW, Kang BN, et al. Piglet gut microbial shifts early in life: causes and effects. J Anim Sci Biotechnol. 2019;10:1-10. doi:10.1186/s40104-018-0308-3.

57. Arfken AM, Frey JF, Summers KL. Temporal dynamics of the gut bacteriome and mycobiome in the weanling pig. Microorganisms. 2020;8:868. doi:10.3390/microorganisms8060868.

58. Rhouma M, Braley C, Th W, Thibodeau A, Quessy S, Fravalo P. Evolution of pig fecal microbiota composition and diversity in response to enterotoxigenic Escherichia coli infection and colistin treatment in weaned piglets. Microorganisms. 2021;7:1459-1474. doi:10.3390/microorganisms9071459.

59. Dou S, Gadonna-Widehem P, Rome V, Hamoudi D, Rhazi L, Lakhal L, et al. Characterisation of earlylife fecal microbiota in susceptible and healthy pigs to post-weaning diarrhoea. PLoS One.2017;12:e0169851. doi:10.1371/journal.pone.0169851.

60. Kim HB, Borewicz K, White BA, Singer RS, Sreevatsan S, Tu ZJ, et al. Microbial shifts in the swine distal gut in response to the treatment with antimicrobial growth promoter, tylosin. Proc Natl Acad 
Sci. 2012;109:15485-15490. doi:10.1073/pnas.1205147109.

61. Unno T, Kim J, Guevarra RB, Nguyen SG. Effects of antibiotic growth promoter and characterization of ecological succession in swine gut microbiota. J Microbiol Biotechnol. 2015;25:431-438. doi:10.4014/jmb.1408.08063.

\section{Tables}

Table 1. Serum metabolites that differed among the dietary treatment groups ${ }^{1}$ 
TRA $^{4}$ vs. REC ${ }^{5}$, d 0 before $E$. coli challenge

\begin{tabular}{|c|c|c|c|}
\hline fructose & 2.13 & 1.88 & 0.108 \\
\hline mannonic acid & 2.21 & 2.01 & 0.083 \\
\hline propyleneglycol & 2.38 & 1.76 & 0.122 \\
\hline \multicolumn{4}{|c|}{$\mathrm{CON}^{6}$ vs. REC, d 5 post-inoculation } \\
\hline mannitol & 0.23 & 1.48 & 0.115 \\
\hline inosine & 0.41 & 1.63 & 0.076 \\
\hline glycerol & 2.09 & 1.79 & 0.045 \\
\hline galactonic acid & 2.26 & 1.65 & 0.076 \\
\hline propyleneglycol & 2.51 & 1.47 & 0.119 \\
\hline shikimic acid & 2.64 & 1.86 & 0.036 \\
\hline \multicolumn{4}{|c|}{ TRA vs. REC, d 5 post-inoculation } \\
\hline 2-hydroxyvaleric acid & 0.24 & 2.13 & 0.001 \\
\hline p-hydroxylphenyllactic acid & 0.30 & 1.10 & 0.145 \\
\hline pipecolinic acid & 0.38 & 1.58 & 0.024 \\
\hline 1-methylhydantoin & 0.40 & 1.51 & 0.031 \\
\hline histidine & 0.45 & 1.98 & 0.002 \\
\hline creatine & 0.46 & 1.51 & 0.031 \\
\hline myo-inositol & 2.01 & 1.93 & 0.002 \\
\hline guanine & 2.03 & 1.83 & 0.005 \\
\hline oleic acid & 2.03 & 1.18 & 0.114 \\
\hline montanic acid & 2.05 & 1.57 & 0.024 \\
\hline galactonic acid & 2.11 & 1.42 & 0.046 \\
\hline hypoxanthine & 2.14 & 1.80 & 0.006 \\
\hline glycerol & 3.26 & 1.35 & 0.067 \\
\hline propyleneglycol & 4.04 & 2.00 & 0.002 \\
\hline shikimic acid & 4.47 & 1.63 & 0.020 \\
\hline taurine & 5.58 & 1.27 & 0.082 \\
\hline
\end{tabular}




\begin{tabular}{llll} 
CON vs. REC, d 11 post-inoculation & & & \\
\hline glycerol & 0.33 & 1.74 & 0.181 \\
\hline inositol-4-monophosphate & 0.48 & 1.92 & 0.165 \\
\hline chenodeoxycholic acid & 3.01 & 1.84 & 0.171 \\
\hline TRA vs. REC, d 11 post-inoculation & & & \\
\hline p-tolyl glucuronide & 0.26 & 2.10 & 0.106 \\
\hline glycerol & 0.30 & 1.84 & 0.195 \\
\hline mannitol & 0.30 & 1.90 & 0.158 \\
\hline 2-ketoisocaproic acid & 0.45 & 1.90 & 0.158 \\
\hline inositol-4-monophosphate & 0.48 & 2.08 & 0.106 \\
\hline chenodeoxycholic acid & 4.67 & 2.04 & 0.109
\end{tabular}

${ }^{1}$ Fold change values less than one indicate that the differential metabolites were reduced in the CON compared to REC or TRA compared to REC, respectively.

${ }^{2} \mathrm{VIP}=$ Variable Importance in the projection.

${ }^{3} \mathrm{FDR}=$ False discovery rate.

${ }^{4} \mathrm{TRA}=$ Trace amounts of antibiotic.

${ }^{5} \mathrm{REC}=$ Label-recommended dose of antibiotic.

${ }^{6} \mathrm{CON}=$ Control.

Table 2. Colon digesta metabolites that differed among the dietary treatment groups ${ }^{1}$ 


\begin{tabular}{llll} 
Metabolite & Fold Change & VIP $^{2}$ & FDR $^{3}$ \\
\hline TRA $^{4}$ vs. REC & & & \\
\hline octadecanol & & & \\
\hline nonadecanoic acid & 0.38 & 1.82 & 0.173 \\
\hline adipic acid & 0.39 & 1.89 & 0.126 \\
\hline pinitol & 0.40 & 1.91 & 0.125 \\
\hline 3-hydroxy-3-methylglutaric acid & 2.22 & 1.82 & 0.173 \\
\hline proline & 2.57 & 1.94 & 0.118 \\
\hline arabitol & 2.64 & 1.92 & 0.118 \\
\hline lyxitol & 3.42 & 2.18 & 0.018 \\
\hline dehydroabietic acid & 3.92 & 2.16 & 0.018 \\
\hline propyleneglycol & 4.27 & 2.15 & 0.018 \\
\hline maltotriose & 5.09 & 1.92 & 0.118 \\
\hline 2-hydroxyvaleric acid & 5.18 & 1.96 & 0.118 \\
\hline
\end{tabular}

TRA vs. REC, d 11 post-inoculation

$\begin{array}{llll}\text { inosine } & 0.20 & 1.9793 & 0.160\end{array}$

${ }^{1}$ Fold change values less than one indicate that the differential metabolites were reduced in the TRA compared to REC.

${ }^{2} \mathrm{VIP}=$ Variable Importance in the projection.

${ }^{3} \mathrm{FDR}=$ False discovery rate.

${ }^{4} \mathrm{TRA}=$ Trace amounts of antibiotic.

${ }^{5} \mathrm{REC}=$ Label-recommended dose of antibiotic.

\section{Figures}


$\mathbf{A}$

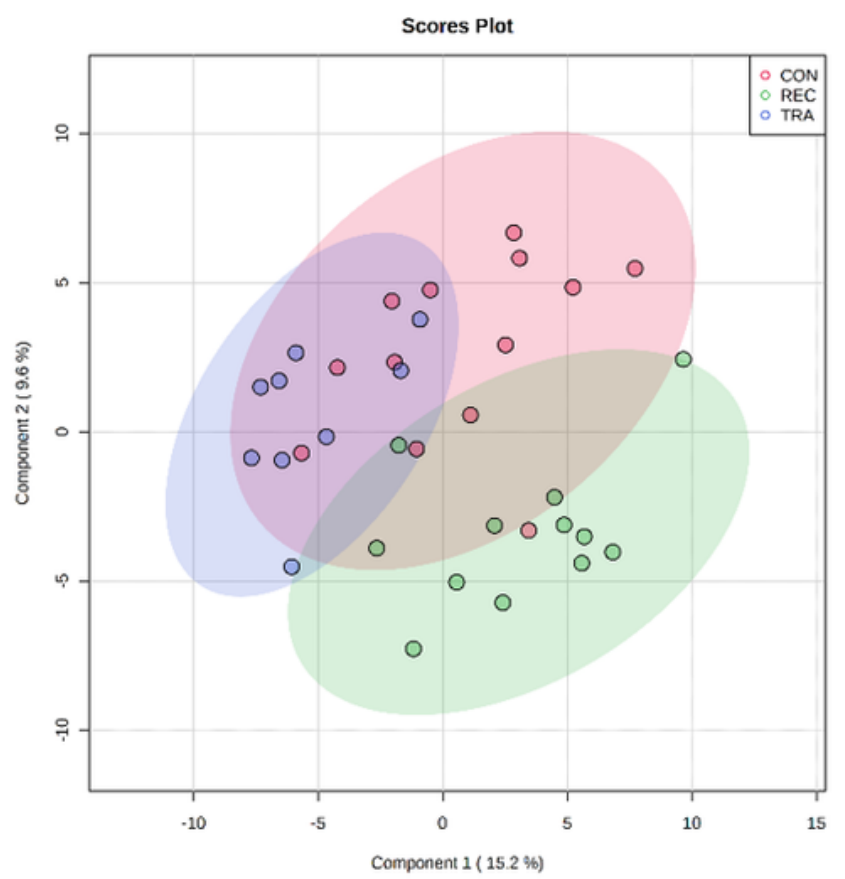

B

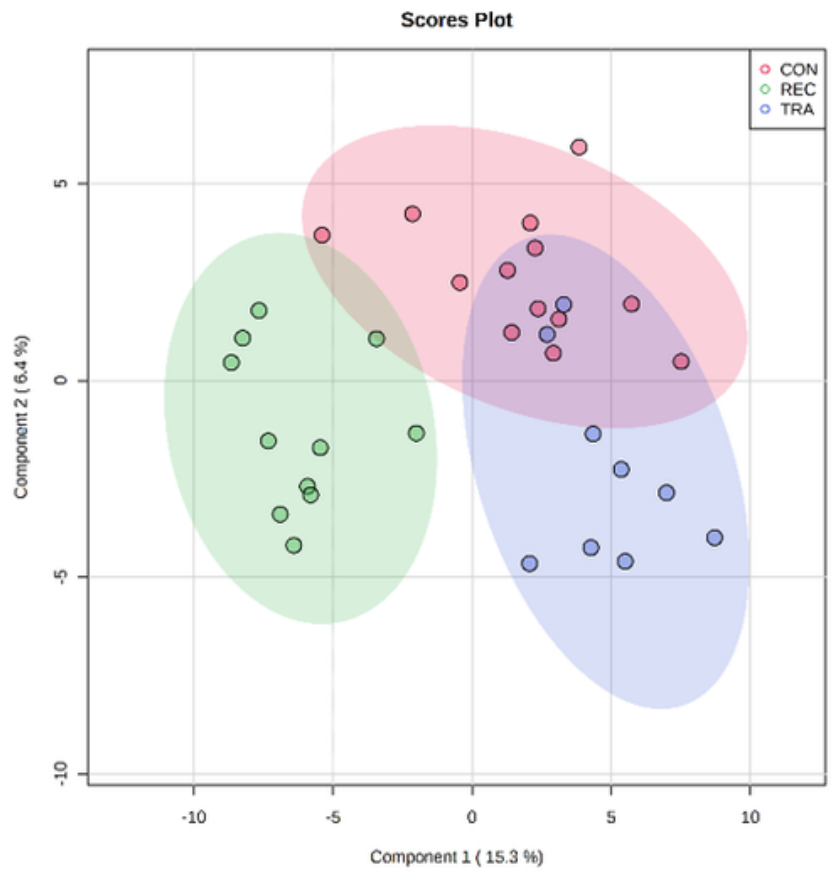

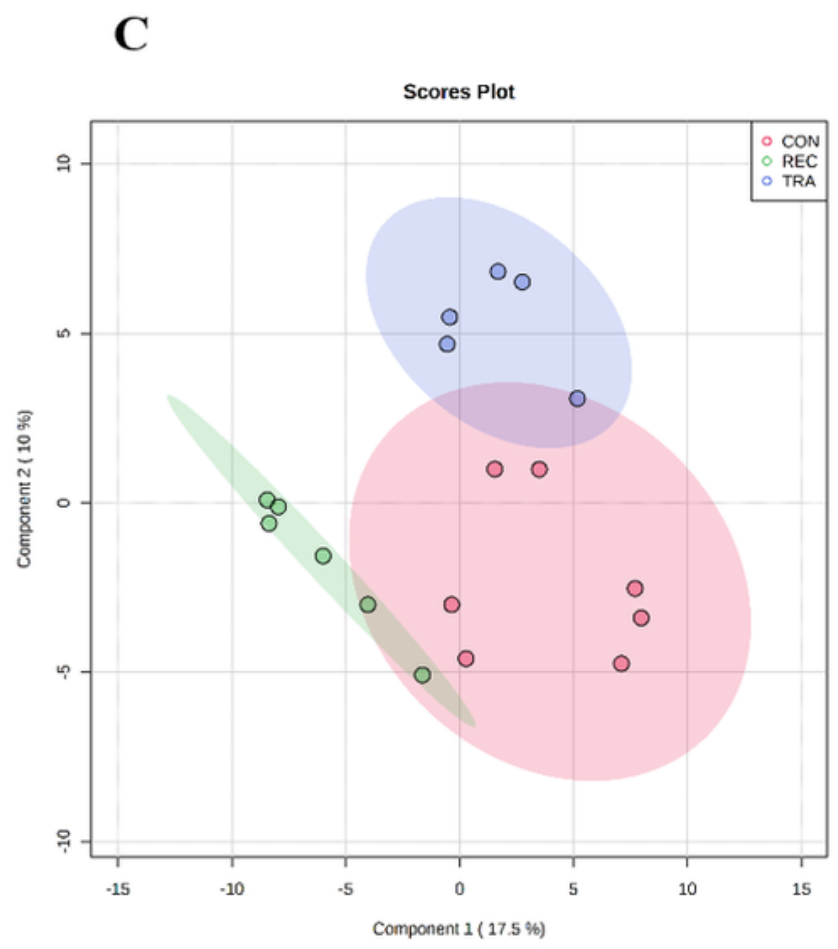

Figure 1

Please see the Manuscript file for the complete figure caption. 

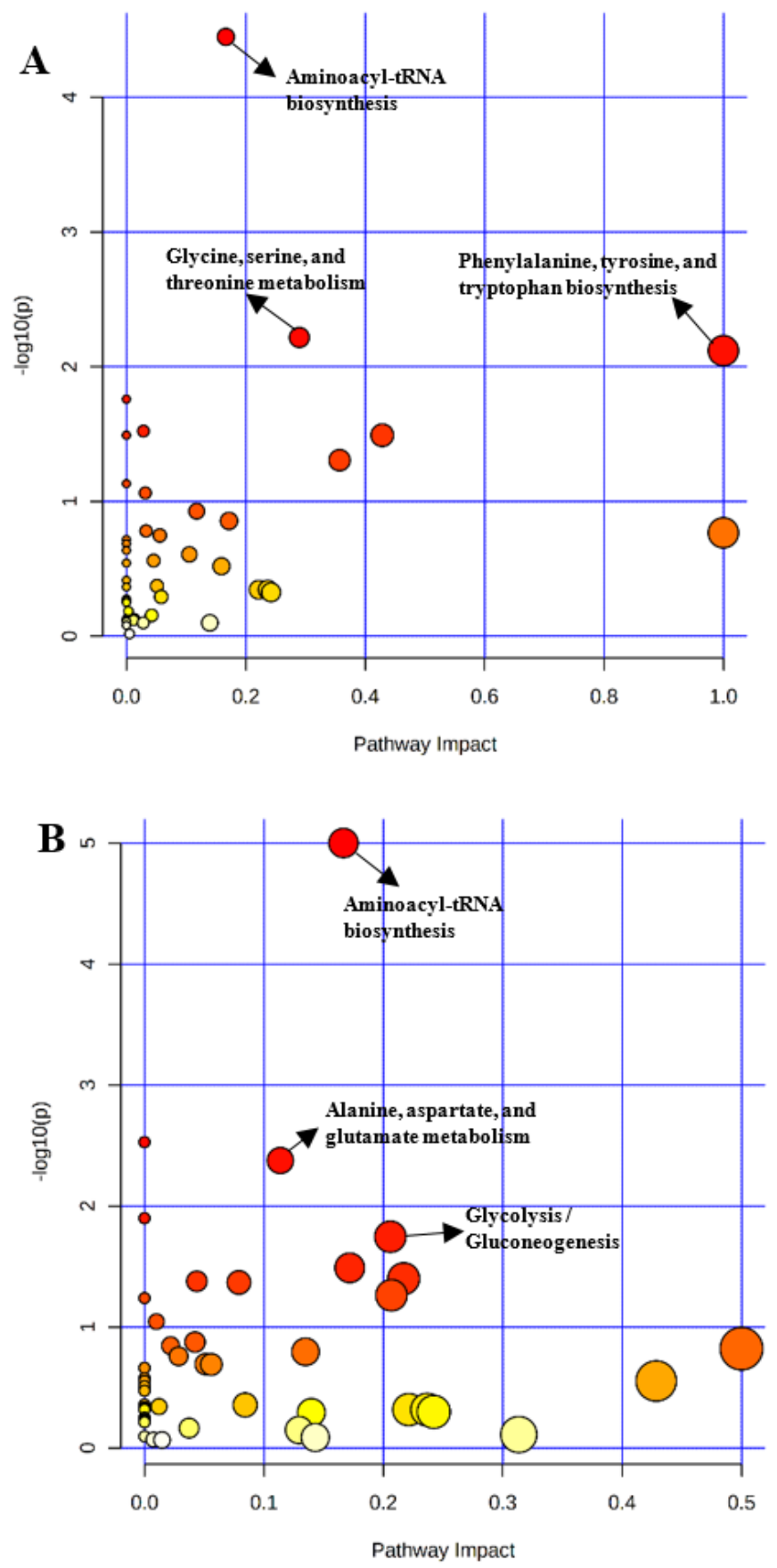

C

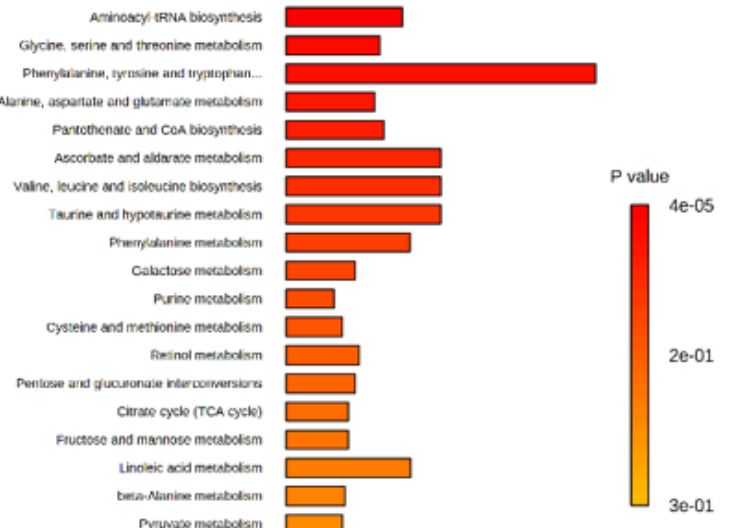

D

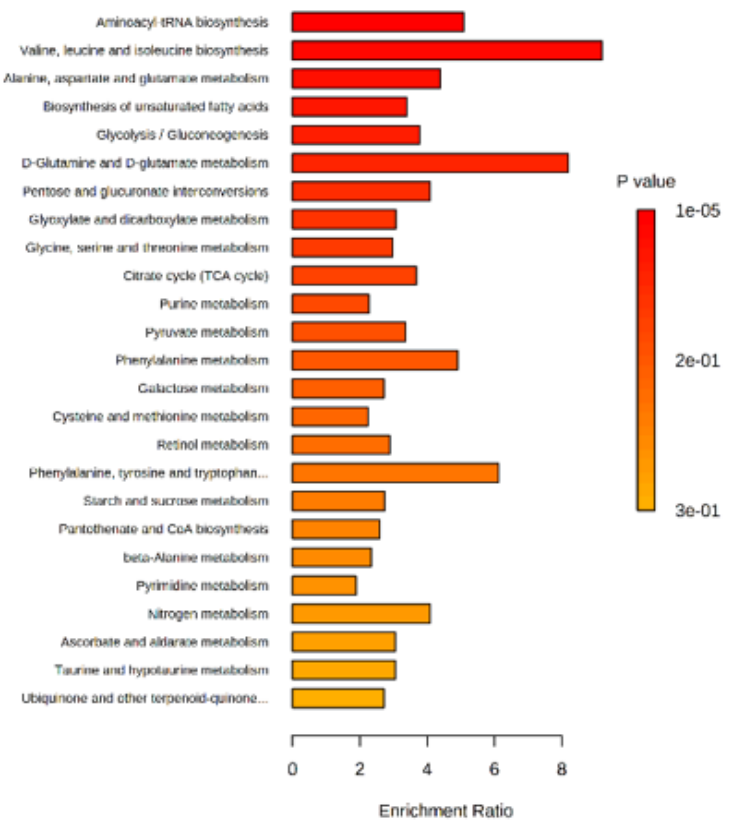

Figure 2

Significantly changed pathways in serum between the control (CON) and label-recommended dose of antibiotic (REC) groups (A), and trace amounts of antibiotic (TRA) and REC groups (B) on d 5 postinoculation. The $x$-axis represents the pathway impact values and the $y$-axis represents the $-\log (P)$ values from the pathway enrichment analysis. Metabolite set enrichment analysis (C, D) shows the metabolic pathways were enriched in CON compared to REC, and TRA compared to REC on d 5 post-inoculation, respectively. Both pathway analysis and metabolite set enrichment analysis were performed using identified metabolites with VIP $>1$. 
$\mathbf{A}$

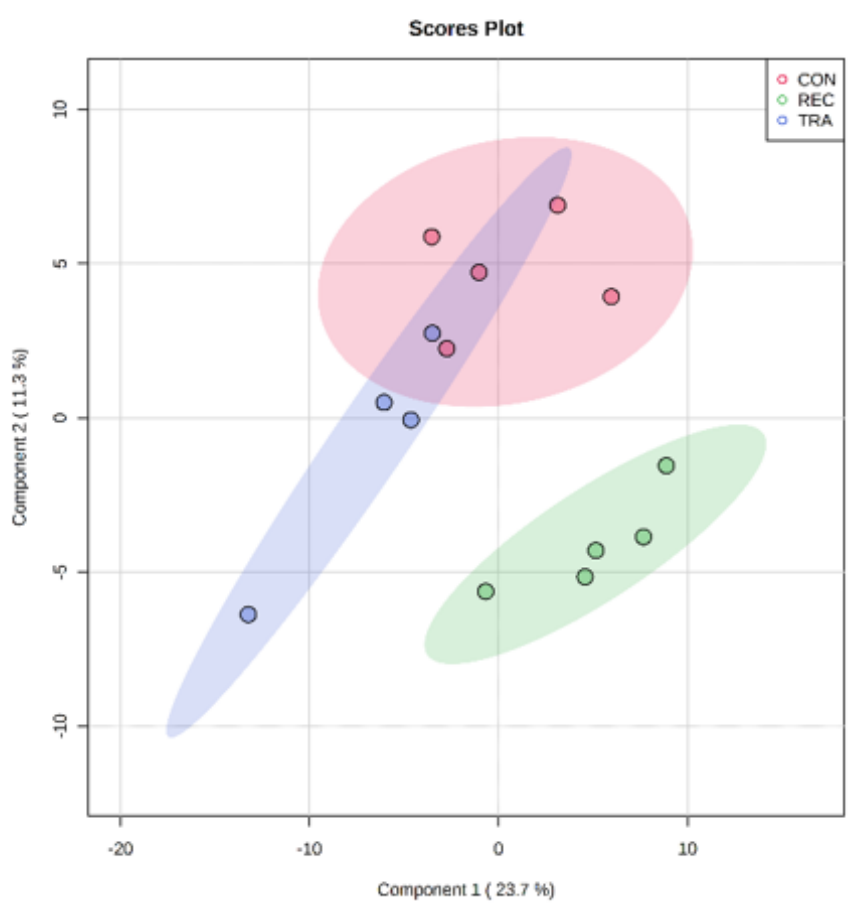

B

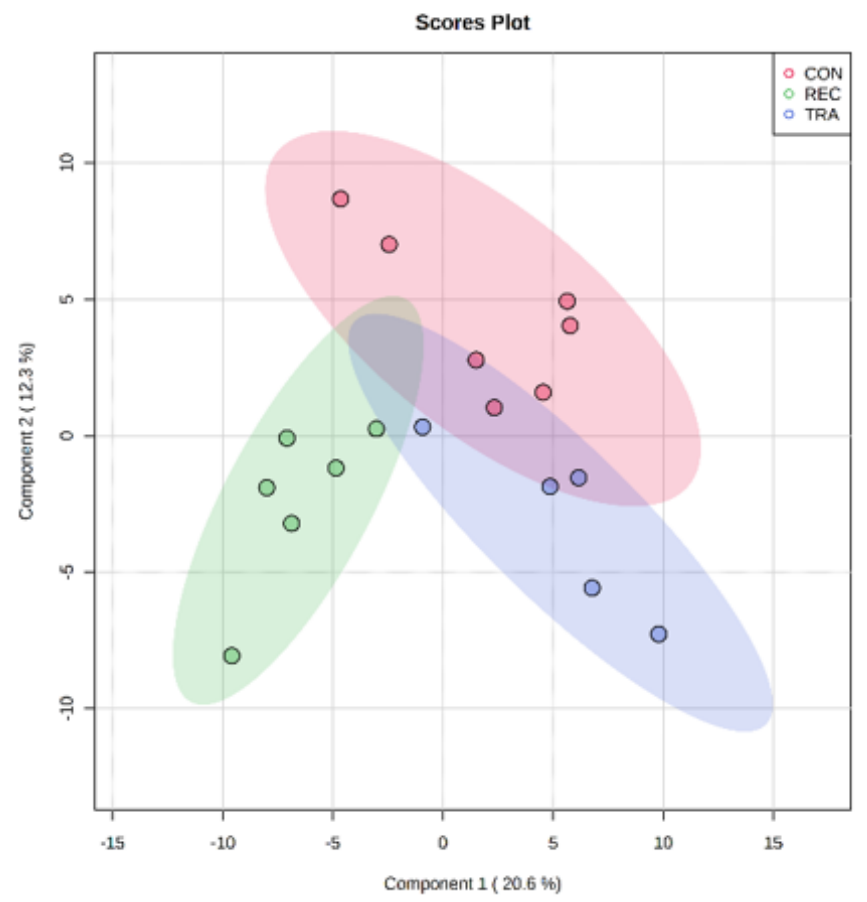

Figure 3

Please see the Manuscript file for the complete figure caption. 

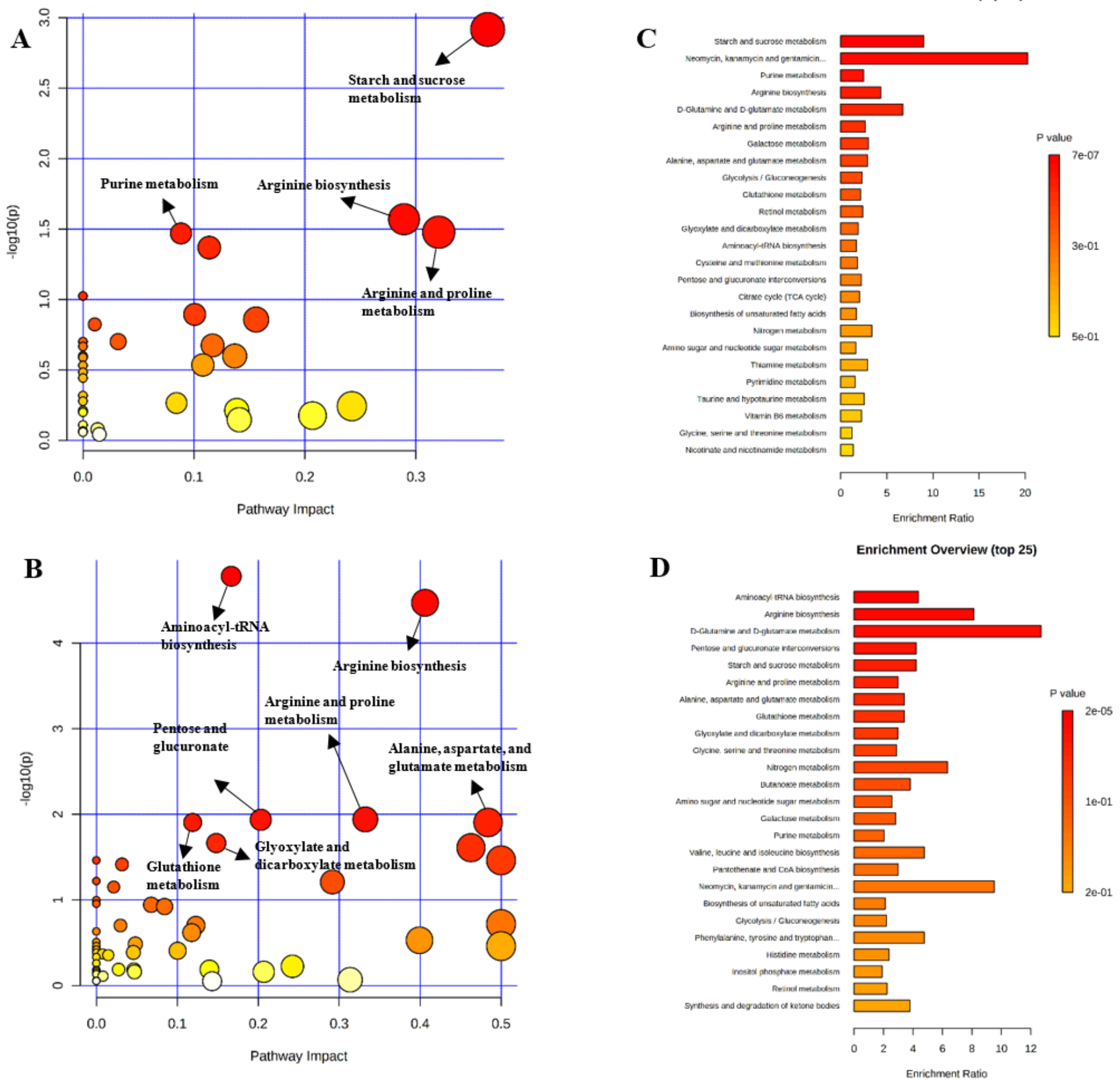

\section{Figure 4}

Significantly changed pathways in colon digesta between the trace amounts of antibiotic (TRA) and label-recommended dose of antibiotic (REC) groups on $\mathrm{d} 5$ (A) and 11 post-inoculation (B). The x-axis represents the pathway impact values and the $y$-axis represents the - $\log (P)$ values from the pathway enrichment analysis. Metabolite set enrichment analysis (C, D) shows the metabolic pathways were enriched in TRA compared to REC group on d 5 and 11 post-inoculation, respectively. Both pathway analysis and metabolite set enrichment analysis were performed using identified metabolites with VIP > 1. 


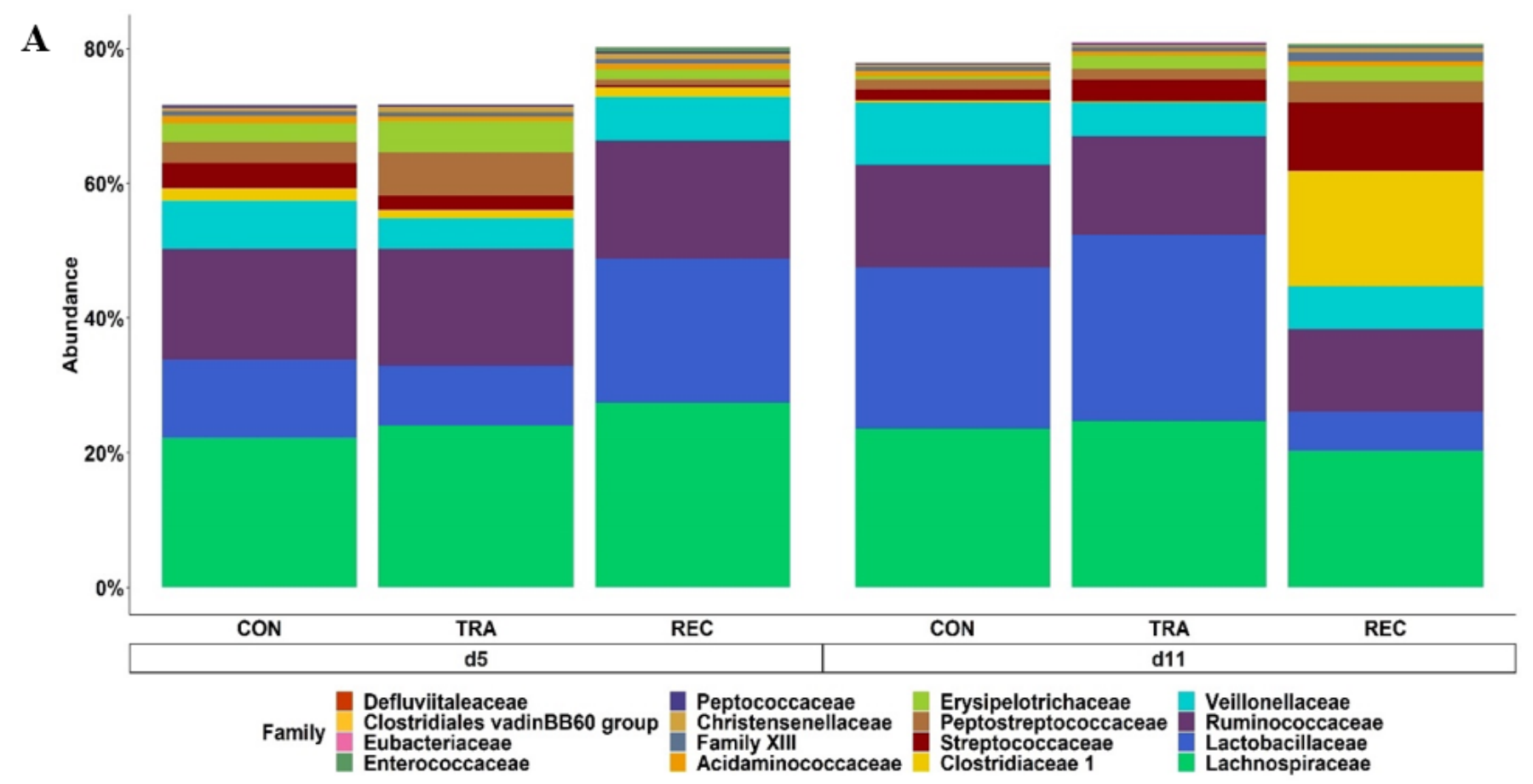

B
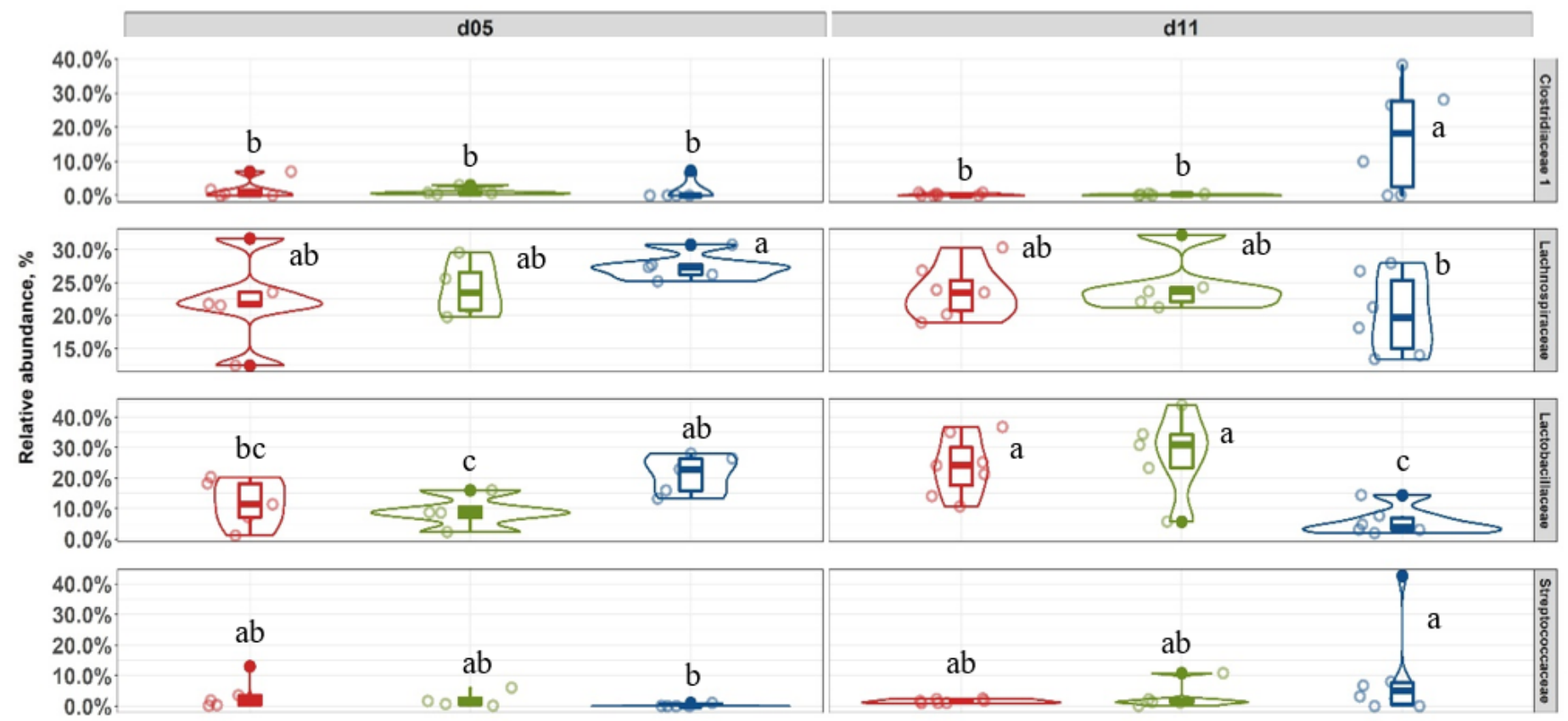

Figure 5

Stacked bar plot showing the relative abundance of Firmicutes family in colon digesta of enterotoxigenic E. coli F18 challenged pigs fed diets supplemented with different dose of antibiotic on d 5 and 11 postinoculation (A). Violin plot showing the relative abundance of individual bacterial phylum (B). a-cMeans without a common superscript are different across both time points (Diet $\times$ day, $P<0.05$ ). Each least squares mean represents 4 to 7 observations. $\mathrm{CON}=$ Control; TRA $=$ Trace amount of antibiotic; $\mathrm{REC}=$ Label-recommended dose of antibiotic. 

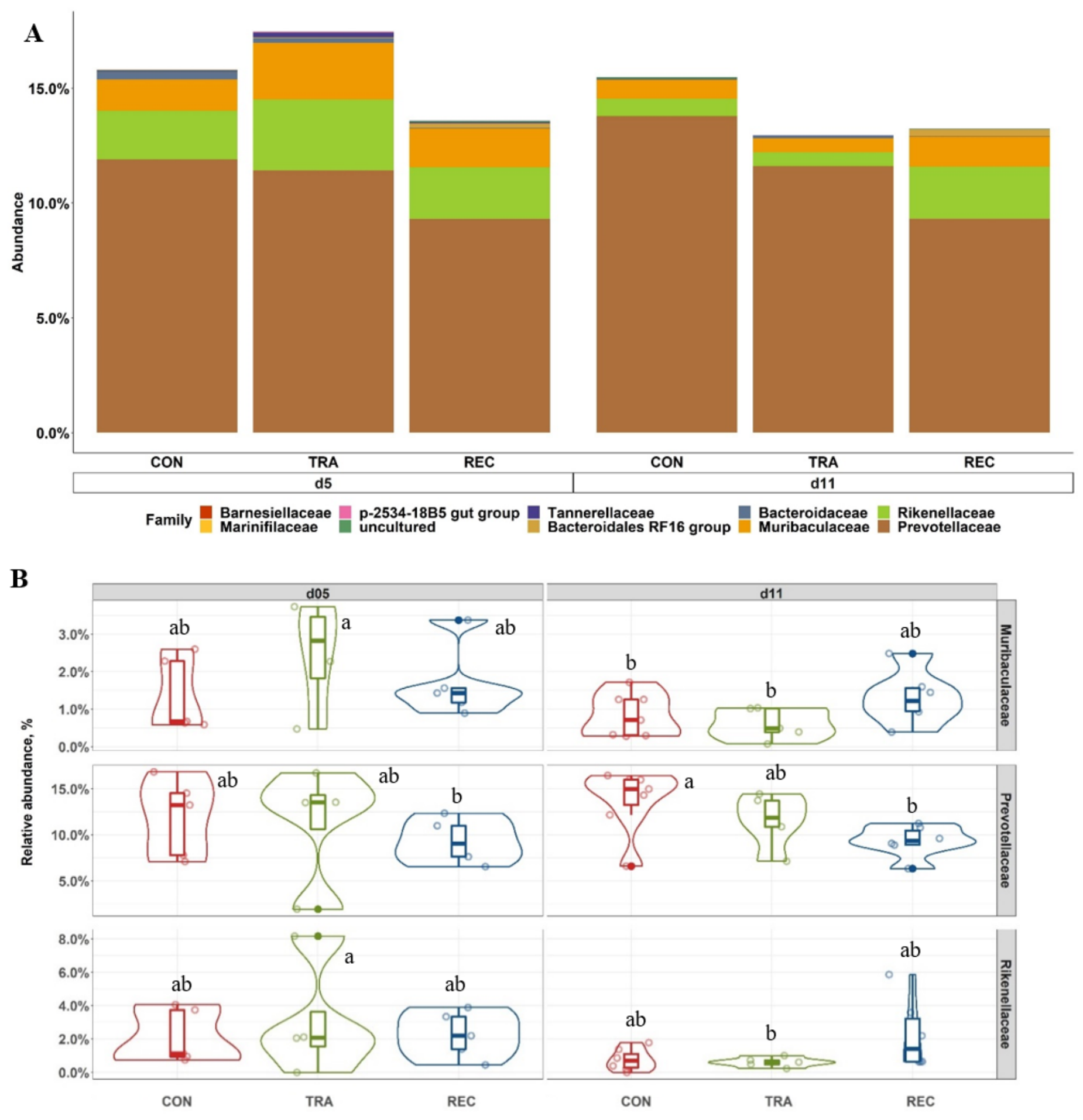

Figure 6

Stacked bar plot showing the relative abundance of Bacteroidetes family in colon digesta of enterotoxigenic E. coli F18 challenged pigs fed diets supplemented with different dose of antibiotic on d 5 and 11 post-inoculation (A). Violin plot showing the relative abundance of individual bacterial phylum (B). a-bMeans without a common superscript are different across both time points (Diet $\times$ day, $P<0.05$ ). Each 
least squares mean represents 4 to 7 observations. $\mathrm{CON}=$ Control; TRA = Trace amount of antibiotic; REC $=$ Label-recommended dose of antibiotic.

\section{Supplementary Files}

This is a list of supplementary files associated with this preprint. Click to download.

- Additionalfile1.docx 